\title{
An additive representation on the product of complete, continuous extensive structures
}

\author{
Yutaka Matsushita
}

Published online: 12 August 2008

(C) Springer Science+Business Media, LLC. 2008

\begin{abstract}
This article develops an axiom system to justify an additive representation for a preference relation $\succsim$ on the product $\prod_{i=1}^{n} A_{i}$ of extensive structures. The axiom system is basically similar to the $n$-component $(n \geq 3)$ additive conjoint structure, but the independence axiom is weakened in the system. That is, the axiom exclusively requires the independence of the order for each of single factors from fixed levels of the other factors. The introduction of a concatenation operation on each factor $A_{i}$ makes it possible to yield a special type of restricted solvability, i.e., additive solvability and the usual cancellation on $\prod_{i=1}^{n} A_{i}$. In addition, the assumption of continuity and completeness for $A_{i}$ implies a stronger type of solvability on $A_{i}$. The additive solvability, cancellation, and stronger solvability axioms allow the weakened independence to be effective enough in constructing the additive representation.
\end{abstract}

Keywords Additive representation · Conjoint structure $\cdot$ Independence $\cdot$ Solvability

\section{Introduction}

Encounter is sometimes made with a choice problem over temporal sequences of positive-valued outcomes. Choices among income streams are taken up as a typical example. In this case, an addition on each time period will be often used to facilitate the evaluation of $n$-tuples of incomes. Let $A^{(n)}=A_{1} \times \cdots \times A_{n}$ be an $n$-component product of nonempty sets of positive-valued outcomes. This article assumes that a concatenation operation $\circ_{i}$ exists on each factor $A_{i}$. To be more precise, $\circ_{i}$ is to be given to form an extensive structure with a view to allowing the addition to be generalized

Y. Matsushita $(\varangle)$

Research Laboratory for Affective Design Engineering, Kanazawa Institute of Technology,

3-1 Yatsukaho, Hakusan, Ishikawa 924-0838, Japan

e-mail: yutaka@neptune.kanazawa-it.ac.jp 
so that psychological or physical attributes will be measured. The purpose of this article is to present axioms adequate enough to assume that $\circ_{i}$ is in existence and is allowed to justify an additive representation of a preference relation $\succsim$ on $A^{(n)}$, i.e., $\left(a_{1}, \ldots, a_{n}\right) \succsim\left(b_{1}, \ldots, b_{n}\right) \Leftrightarrow \sum_{i=1}^{n} u_{i}\left(a_{i}\right) \geq \sum_{i=1}^{n} u_{i}\left(b_{i}\right)$, where $u_{i}$ is a real-valued function on $A_{i}$ that represents $\circ_{i}$ additively, i.e., $u_{i}\left(a_{i} \circ_{i} b_{i}\right)=u_{i}\left(a_{i}\right)+u_{i}\left(b_{i}\right)$.

The additive representation on $A^{(n)}$ is similarly axiomatized to conjoint measurement (Krantz et al. 1971). Therefore, the monotonicity axiom, which is essential to theories (Fishburn 1982, Theorems 9.1 and 9.2; Wakker 1989, Theorem A2.1) where premise is made on a componentwise operation on product sets, is found out to be unnecessary on $A^{(n)}$. An axiom expressed as a property of multifactor, such as the monotonicity axiom, will further be expelled. It is widely known that there are two types of independence; one is a stronger one, and another is a weaker one. Independence of a stronger type requires independence of the order for any of the two factors or more from the fixed levels of the other factors. In this connection, a weaker one means independence of the order with respect to each single factor. Krantz et al. (1971) used the independence of a stronger type for the $n$-component $(n \geq 3)$ additive conjoint structure, by which the ordering of the "joint effect" of multiple factors is forced to be considered to examine the validity. This article will be subservient enough to develop an axiom system having the independence of a weaker type to be utilized for construction of the additive representation. At the same time, it is stipulated that restricted solvability should be used in this axiom system. Just a slight amount of development was already made by Matsushita (2007) with an axiom system having the independence of a weaker type. But the proposed axiom system is never encouraged to be a weaker version of the $n$-component additive conjoint structure. This is because it has unrestricted solvability although the conjoint structure has restricted solvability and because the latter is known as the one much weaker than the former. In summary, the present article finds itself in a position as a revised version ${ }^{1}$ of Matsushita (2007) in the sense that a practical axiom system will be proposed not less than the $n$-component additive conjoint structure.

The construction of the additive representation referred to above is the one similar to that of Krantz et al. with the exception that use of independence is a different one. That is, a major role will be given to independence thanks to the approach in this article in constructing the additive representation on a bounded set. On the other hand, Krantz etal. made use of independence not only for the construction on a bounded set but also for the extension of the representation to the whole set (Remark2). Usefulness is noticed with the assumption of $o_{i}$ existing on $A_{i}$ and validity of solvability of a stronger type ${ }^{2}$ in applying the independence of a weaker type. To guarantee the solvability of a stronger type, it is further assumed that $A_{i}$ is continuous and Dedekind complete and that $A_{i}$ has no minimal positive element. However, these assumptions seem rational as long as the measurement object in question is confined to quantities

\footnotetext{
1 Matsushita (2007) restricted an operation on extensive structures to a "closed" binary operation, but the present article does not. Also in this respect, the present article is more general than the previous one.

2 Krantz et al. (1990) called it "solvability" simply and solvability of a weaker type in this article "restricted solvability." However, we will use the term restricted solvability for a solvability axiom on product sets.
} 
of the same infinitely divisible good, such as amounts of money (Marchant and Luce 2003) or intensities of pure tones (Luce 2004).

The reminder of this article is comprised of the following sections. Section 2: This reviews the extensive structure and its representation, including the concepts of completeness and semicontinuity. In particular, it is shown that implication is made with the solvability of a stronger type by completeness and lower semicontinuity. Section 3: This presents axioms. In the axioms, restricted solvability of a special type-additive solvability - and the usual cancellation are dealt with as unique axioms with a view to constructing the additive representation on $A^{(n)}$. Also, a brief comment is made on a restriction that arises when each factor of the conjoint structure is extensive. Section 4: Conclusions are provided in this section. Section 5: The proofs of the proposition and theorem along with the preliminary lemmas are handled in this section.

\section{Complete, continuous extensive structures}

Throughout this article, $\mathbb{Z}^{+}, \mathbb{Z}_{0}^{+}$, and $\mathbb{R}_{0}^{+}$denote the sets of all positive integers, all non-negative integers, and all non-negative real numbers, respectively. Further, let $\succsim$ be a binary relation on a nonempty set $A$ that is interpreted as a preference relation. As usual, $\succ$ denotes the asymmetric part, $\sim$ the symmetric part, and $\precsim, \prec$ denote reversed relations. The binary relation $\succsim$ on $A$ is a weak order if and only if it is connected and transitive. An element $e \in A$ denotes no change of the status quo. Let $\circ$ be a "partial" binary operation on $A$. The operation means a function from a subset $B$ of $A \times A$ into $A$. The expression $a \circ b$ is said to be defined if and only if $(a, b) \in B$. Thus, the attention should be paid to the definition of the concatenation of $n$ copies of an element $a$, denoted $n a$. The sequence $\{n a\}$ is defined inductively by $0 a=e, 1 a=a$, and

$$
n a=(n-1) a \circ a \text { if the right-hand is defined }
$$

$n a$ is undefined otherwise.

The quadruple $\langle A, \succsim, \circ, e\rangle$ is an extensive structure with identity if and only if the following conditions hold for all $a, b, c \in A$ for which the indicated operations $\circ$ are defined:

A1. Weak order: $\succsim$ is a weak order.

A2. Local definability: if $a \succsim c, b \succsim d$, then $c \circ d$ is defined.

A3. Monotonicity: $a \succsim b \Leftrightarrow a \circ c \succsim b \circ c \Leftrightarrow c \circ a \succsim c \circ b$.

A4. Weak associativity: $(a \circ b) \circ c \sim a \circ(b \circ c)$.

A5. Solvability (of a weaker type): whenever $a \succ b$, there exists $c \in A$ such that $a \succsim b \circ c \succ b$.

A6. Identity: $e \circ a \sim a \circ e \sim a$.

A7. Positivity: $a \circ b \succ b$ unless $a \sim e$.

A8. Archimedean: every bounded sequence $\{n a\}$ is finite.

Note that A6 is not included in the definition of an extensive structure given by Krantz et al. (1971, 1990). In an extensive structure with identity, A7 is satisfied if and 
only if $a \succ e$. An element $a$ of $A$ such that $a \succ e$ is called positive. In particular, the triple $\langle A, \succsim$, o $\rangle$ satisfying $\mathrm{A} 1-\mathrm{A} 3$ is said to be a concatenation structure.

The stronger version of A5 is written as follows:

$\mathbf{A 5}^{\prime}$. Solvability of a stronger type: whenever $a \succ b$, there exists $c \in A$ such that $b \circ c$ is defined and $a \sim b \circ c$.

Fuchs (1963) adopted A5' with a view to constructing an order-preserving additive function on an extensive structure with $\circ$ restricted to a "closed" binary operation, i.e., $a \circ b \in A$ for all $a, b \in A$. On the other hand, Roberts and Luce (1968) developed a more practical axiom system including none of $\mathrm{A} 5^{\prime}$. Let it hereby be understood that quantities, which are of interest in this article, are a homogeneous substance or a certain of continuum. It may therefore be easy to provide properties on extensive structures from which $\mathrm{A} 5^{\prime}$ can be derived. In finalizing this section, such properties are considered for the purpose of utilizing $\mathrm{A}^{\prime}$ in extending the additive representation from a bounded domain to the whole domain. Hereafter, the case where there is no minimal positive element will exclusively be dealt with. The trivial case where $A$ has just a single element $e$ will always be excluded. As a matter of course, introduction is made with the following two properties. A concatenation structure $\langle A, \succsim, \circ\rangle$ is Dedekind complete (for short, complete) if and only if every nonempty subset of $A$ that has an upper bound has a least upper bound in $A .\langle A, \succsim, 0\rangle$ is continuous if and only if $\circ$ is continuous as a function of two variables, using the order topology on its range and the relative product order topology on its domain. ${ }^{3}$ The definition shown below (Krantz et al. 1990) is more concrete than the definition of continuity.

Definition 1 Let $\langle A, \succsim$, ○ $\rangle$ be a concatenation structure satisfying A7 and having no minimal positive element. The structure $A$ is said to be lower semicontinuous (related to left-concatenation by $a \in A$ ) if and only if whenever $a \circ b$ is defined and $\succ c$, then there exists $b^{\prime} \in A$ such that $b \succ b^{\prime}$ and $a \circ b^{\prime} \succ c$. A is upper semicontinuous (related to left-concatenation by $a$ ) if and only if whenever $a \circ b$ is defined and $\prec c$, and $a \circ b^{\prime \prime}$, with $b^{\prime \prime} \succ b$, is also defined, then there exists $b^{\prime} \in A$ such that $b^{\prime \prime} \succsim b^{\prime} \succ b$ and $c \succ a \circ b^{\prime}$.

The definition of upper semicontinuity is more complicated than that of lower because when $c \succ a \circ b$, some additional condition is needed to assert that there is a larger $b^{\prime} \succ b$ such that $a \circ b^{\prime}$ is defined. But in the case of lower semicontinuity, no corresponding problem arises because of local definability, A2, and the assumption of $A$ having no minimal positive element. In an extensive structure, $\circ$ is proved to be weakly commutative: whenever, $a \circ b$ is defined, then $a \circ b \sim b \circ a$. Therefore, if $A$ is lower or upper semicontinuous related to "left-concatenation", then so it is in the "right-concatenation" case. The next result following from the proof of Theorem 19.6 (Krantz et al. 1990) is quite a useful one for the verification of continuity: For any extensive structure with no minimal positive element, the structure is continuous if and only if it is both lower and upper semicontinuous.

Proposition 1 Let $\langle A, \succsim$, o, e $\rangle$ be an extensive structure with no minimal positive element. Then

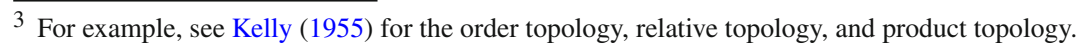


(i) A is upper semicontinuous.

(ii) Completeness and lower semicontinuity imply $A 5^{\prime}$.

Definition 2 A complete, continuous extensive structure $\langle A, \succsim, \circ, e\rangle$ is an extensive structure with identity that is complete and lower semicontinuous.

The following representation theorem is the fundamentals of the additive representation in this article.

Theorem 1 Let $\langle A, \succsim, \circ$, e $\rangle$ be a complete, continuous extensive structure with no minimal positive element. Then there exists a function $u$ from A into $\mathbb{R}_{0}^{+}$such that for all $a, b \in A$,

$$
\begin{aligned}
& a \succsim b \Leftrightarrow u(a) \geq u(b) \\
& u(a \circ b)=u(a)+u(b) \text { whenever } a \circ b \text { is defined; } \\
& u(e)=0,
\end{aligned}
$$

where $u$ is continuous ${ }^{4}$ using the order topology of $A$ and the relative topology of $u(A)$. Moreover, this representation is unique up to multiplication by a positive constant.

An additive representation on $A$ is a real-valued function satisfying the orderpreserving (Eq. 1) and additivity (Eq. 2) properties of Theorem 1. For the sake of clarification, the term "additive representation" is used to imply an order-preserving function $U$ on the Cartesian product of nonempty sets $A_{i}$; the function $U$ is written as $U\left(a_{1}, \ldots, a_{n}\right)=\sum_{i=1}^{n} u_{i}\left(a_{i}\right)$, where $u_{i}$ is a real-valued function on $A_{i}$.

Remark 1 From the comment before Theorem 19.7 of Krantz et al. (1990), it is seen that if the extensive structure $A$ of Theorem 1 has a maximal element, then $u(A)$ equals a bounded closed interval $[0, r]$ where $r>0$. Otherwise, it is determined that $u(A)=\mathbb{R}_{0}^{+}$. Emphasis should be attached onto the case of $u(A)=[0, r]$ rather than the case of $u(A)=\mathbb{R}_{0}^{+}$, because restricted solvability is essential to the former case in conjoint measurement.

If, in Theorem 1, $A$ is the set of amounts of money, i.e., $A=\mathbb{R}_{0}^{+}$, and $a \circ b=a+b$ for $a, b \in \mathbb{R}_{0}^{+}$, then it is understood that the additive representation is proportional to money, i.e., $u(a)=\alpha a, \alpha>0$. This fact is contradictory to individual evidences of diminishing marginal utility for gains. To solve this problem, Marchant and Luce (2003) proposed addition rules different from $a \circ b=a+b$ for $a, b \in \mathbb{R}_{0}^{+}$, and obtained for $u(a)$ more flexible forms than the linear one. In addition, they utilized the concept of "joint receipt" (Luce 1990, 2000) $)^{5}$ to interpret the addition rules. In view of establishing the property of diminishing marginal utility, it will be rational to consider $u$ bounded above. To begin with, it may be suitable in actual decision-making problems to assume income of each period to be bounded above.

\footnotetext{
4 See Candeal et al. (1996) for the axiomatization of a continuous additive representation on an extensive structure with o restricted to a closed binary operation.

5 A Joint receipt operation is a closed binary operation on an extensive structure with identity. Luce (2000) used the operation to express a simultaneous receipt of gambles (including amounts of money) and showed some types of representations and applications.
} 


\section{Additive representation on the product}

Let $N=\{1, \ldots, n\}, n \geq 2$. Throughout the article, for each $i \in N$, let $\left\langle A_{i}, \succsim_{i}\right.$, $\left.\circ_{i}, e_{i}\right\rangle$ be a complete, continuous extensive structure with no minimal positive element. Let $A^{(n)}=\prod_{i=1}^{n} A_{i}$ be the Cartesian product of $A_{i}$. The following are used as notation. For $a=\left(a_{1}, \ldots, a_{n}\right) \in A^{(n)}, a_{\bar{i}}$ and $a_{\overline{i j}}$ denote that the $i$-th component and the $i$ - and $j$-th components of $a$ are omitted, respectively:

$$
\begin{aligned}
a_{\bar{i}} & =\left(a_{1}, \ldots, a_{i-1}, a_{i+1}, \ldots, a_{n}\right), \\
a_{\overline{i j}} & =\left(a_{1}, \ldots, a_{i-1}, a_{i+1}, \ldots, a_{j-1}, a_{j+1}, \ldots, a_{n}\right) .
\end{aligned}
$$

Let $\left(b_{i}, a_{\bar{i}}\right)$ and $\left(b_{i}, b_{j}, a_{\overline{i j}}\right)$ denote the replacement of $a_{i}$ by $b_{i}$ and that of $a_{i}, a_{j}$ by $b_{i}, b_{j}$, respectively:

$$
\begin{aligned}
\left(b_{i}, a_{\bar{i}}\right) & =\left(a_{1}, \ldots, a_{i-1}, b_{i}, a_{i+1}, \ldots, a_{n}\right), \\
\left(b_{i}, b_{j}, a_{\overline{i j}}\right) & =\left(a_{1}, \ldots, a_{i-1}, b_{i}, a_{i+1}, \ldots, a_{j-1}, b_{j}, a_{j+1}, \ldots, a_{n}\right) .
\end{aligned}
$$

The set of all such elements $a_{\bar{i}}$ is denoted by $A_{\bar{i}}^{(n)}$. The symbol $\circ_{\bar{i}}$ is used to indicate $a_{\bar{i}} \circ_{\bar{i}} c_{\bar{i}}=\left(a_{1} \circ_{1} c_{1}, \ldots, a_{i-1} \circ_{i-1} c_{i-1}, a_{i+1} \circ_{i+1} c_{i+1}, \ldots, a_{n} \circ_{n} c_{n}\right)$, and $\left(a_{1} \circ_{1} c_{1}, \ldots, a_{n} \circ_{n} c_{n}\right)$ is often written as $\left(a_{i} \circ_{i} c_{i}, a_{\bar{i}} \circ_{\bar{i}} c_{\bar{i}}\right)$. Also, let $k_{\bar{i}} a_{\bar{i}}=$ $\left(k_{1} a_{1}, \ldots, k_{i-1} a_{i-1}, k_{i+1} a_{i+1}, \ldots, k_{n} a_{n}\right)$ for $k_{i} \in \mathbb{Z}_{0}^{+}$, and $\left(k_{1} a_{1}, \ldots, k_{n} a_{n}\right)=\left(k_{i} a_{i}\right.$, $\left.k_{\bar{i}} a_{\bar{i}}\right)$.

For a binary relation $\succsim$ on $A^{(n)}$, two axioms that are similar to those of the conjoint structure are provided. However, the independence axiom is the weaker type: $\succsim$ on $A^{(n)}$ is single-factor independent if and only if for every $i \in N, a_{i} \succsim_{i} b_{i} \Leftrightarrow$ $\left(a_{i}, a_{\bar{i}}\right) \succsim\left(b_{i}, a_{\bar{i}}\right)$ for all $a_{i}, b_{i} \in A_{i}$ and all $a_{\bar{i}} \in A_{\bar{i}}^{(n)}$. This axiom states the relation of the ordering of each extensive structure, $A_{i}$, to that of the product, $A^{(n)}$. In addition, the solvability axiom is formed as combination of "restricted solvability" with the concept of additive decomposability.

Definition 3 A binary relation $\succsim$ on $A^{(n)}$ is additively solvable if and only if for each $i \in N$, whenever $\left(\bar{b}_{i}, b_{\bar{i}}\right) \succsim\left(a_{i}, a_{\bar{i}}\right) \succsim\left(\underline{b}_{i}, b_{\bar{i}}\right)$, then there exists an element $x_{i} \in A_{i}$ that is determined to be independent of $a_{i}$ such that

$$
\left(a_{i} \circ_{i} x_{i}, b_{\bar{i}}\right) \sim\left(a_{i}, a_{\bar{i}}\right) .
$$

The solution is undoubtedly decomposed into the addition of the term $a_{i}$ of the $i$-th component and into the term $x_{i}$ dependent on the other components.

Definition 4 Let $S^{(n)}$ be a subset of $A^{(n)}$ that is endowed with the restriction of $\succsim$, which is also denoted by the same symbol. $\left\langle S^{(n)}, \succsim\right\rangle$ is a symmetrically bounded set from above if and only if there are elements $\bar{b}_{i} \succ_{i} e_{i}$ in $A_{i}$, with $n \bar{b}_{i}$ defined, such that $\left(\bar{b}_{i}, e_{\bar{i}}\right) \sim\left(\bar{b}_{j}, e_{\bar{j}}\right)(i \neq j)$ and for $a_{i}$ in $\left(a_{1}, \ldots, a_{n}\right) \in S^{(n)}, \bar{b}_{i} \succsim_{i} a_{i}$.

The usual cancellation axiom is hereby given for the purpose of constructing the additive representation, utilizing restricted solvability. As a matter of reality, this sort 
of axiom is required for extension of additive representation from $S^{(n)}$ to $A^{(n)}$. The symbol $S^{(n)}$ shall hereafter be used to indicate a symmetrically bounded set from above.

Definition 5 A binary relation $\succsim$ on $A^{(n)}$ satisfies cancellation if and only if for any $\left(a_{i}, a_{\bar{i}}\right),\left(b_{i}, b_{\bar{i}}\right) \in A^{(n)}$ and any $\left(c_{i}, c_{\bar{i}}^{-}\right) \in S^{(n)},\left(a_{i} \circ_{i} c_{i}, a_{\bar{i}} \circ_{\bar{i}} c_{\bar{i}}\right) \sim\left(b_{i} \circ_{i} c_{i}, b_{\bar{i}} \circ_{\bar{i}} c_{\bar{i}}\right)$ implies $\left(a_{i}, a_{\bar{i}}\right) \sim\left(b_{i}, b_{\bar{i}}\right)$.

In the case of two factors, cancellation is written as

$$
\left(a_{1} \circ_{1} c_{1}, a_{2} \circ_{2} c_{2}\right) \sim\left(b_{1} \circ_{1} c_{1}, b_{2} \circ_{2} c_{2}\right) \text { implies }\left(a_{1}, a_{2}\right) \sim\left(b_{1}, b_{2}\right)
$$

for any $\left(a_{1}, a_{2}\right),\left(b_{1}, b_{2}\right) \in A^{(2)}$ and any $\left(c_{1}, c_{2}\right) \in S^{(2)}$. Let $a_{i}, b_{i}$, and $c_{i}$ be amounts of money or lotteries (e.g., incentive wages) to be received in period $i=1,2$. Then, cancellation is interpreted as the disregard of a common bonus for each time period. Kahneman and Tversky (1979) pointed out the disregard may produce inconsistent preferences, because a pair of alternatives can be decomposed into common and distinct components in more than one way, and different decompositions sometimes lead to different preferences (called isolation effect). However, in consideration that all alternatives to be edited including common bonuses are positive, ways of decomposition themselves are restricted. In addition, special attention should be paid to the fact that the cancellation axiom coupled with weak ordering and single-factor independence is successful enough to hold for "any $\left(c_{i}, c_{\dot{i}}\right) \in A^{(n)}$ " in Definition 5.

Remark 2 The Thomsen condition, which is somewhat artificial as the cancellation axiom, is provided for the 2-component additive conjoint structure. However, this condition disappears from the $n$-component $(n \geq 3)$ additive conjoint structure. In fact, the Thomsen condition is derived from the independence of a stronger type and restricted solvability (Krantz et al. 1971, Lemma 6.14). It would be paradoxically expected that the independence of a stronger type is so strong. Despite the above, cancellation is a restricted axiom in comparison with the $n$-component additive conjoint structure.

Definition 6 Assume that each $A_{i}$ is a complete, continuous extensive structure with no minimal positive element. Let $\succsim$ be a binary relation on $A^{(n)}, n \geq 2$. The couple $\left\langle A^{(n)}, \succsim\right\rangle$ is an additive conjoint structure on the product of extensive structures if and only if the following axioms are satisfied:

1. $\succsim$ is a weak order.

2. $\succsim$ is single-factor independent.

3. $\succsim$ satisfies additive solvability.

4. $\succsim$ satisfies cancellation.

It is noted in Definition 6 that $A^{(n)}$ also has no minimal positive element.

Theorem 2 Assume that $\left\langle A^{(n)}, \succsim\right\rangle$ is an additive conjoint structure on the product of extensive structures. Then there exist additive representations $u_{i}$ on $A_{i}$ (Theorem 1), 
any two of which are related by $u_{i}\left(a_{i}\right)=u_{j}\left(a_{j}\right)$ whenever $\left(a_{i}, e_{\bar{i}}\right) \sim\left(a_{j}, e_{\bar{j}}\right)(i \neq j)$ such that for all $a, b \in A^{(n)}$,

$$
a \succsim b \Leftrightarrow \sum_{i=1}^{n} u_{i}\left(a_{i}\right) \geq \sum_{i=1}^{n} u_{i}\left(b_{i}\right)
$$

Moreover, utilities $v_{i}$ on $A_{i}$ satisfy this representation in place of utilities $u_{i}$ if and only if there exists a real number $\alpha>0$ such that for each $i=1, \ldots, n, v_{i}=\alpha u_{i}$.

Remark 3 Let $U\left(a_{1}, \ldots, a_{n}\right)=\sum_{i=1}^{n} u_{i}\left(a_{i}\right)$. Since each $u_{i}$ is an additive representation on the extensive structure $A_{i}$, the following expression is satisfied:

$$
U\left(a_{1} \circ_{1} b_{1}, \ldots, a_{n} \circ_{n} b_{n}\right)=\sum_{i=1}^{n} u_{i}\left(a_{i}\right)+\sum_{i=1}^{n} u_{i}\left(b_{i}\right) .
$$

Theorem 2 states that the additive representation on the conjoint structure can be constructed in terms of extensive measures $u_{i}$. Attention should be paid here to the fact that these extensive measures must be incorporated in the context of conjoint measurement. Care must be taken that each extensive measure is unique up to the similarity transformations, $u_{i} \rightarrow \alpha u_{i}, \alpha>0$, i.e., that each measure is allowed to have a free unit. Therefore, in order to evaluate trade-offs between components, it is essential to unify calibrations of these extensive measures. To comply with the above, the constraint that $u_{i}\left(a_{i}\right)=u_{j}\left(a_{j}\right)$ whenever $\left(a_{i}, e_{\bar{i}}\right) \sim\left(a_{j}, e_{\bar{j}}\right)(i \neq j)$ is provided in Theorem 2. Moreover, the constraint imposes on a tight restriction on behavior of components. Now, assume that $k a_{i}, k a_{j}$ are defined for $k \in \mathbb{Z}^{+}$and that $\left(a_{i}, e_{\bar{i}}\right) \sim\left(a_{j}, e_{\bar{j}}\right)$. Then from the equation $u_{i}\left(k a_{i}\right)=u_{j}\left(k a_{j}\right)$ (which is implied by $\left.u_{i}\left(a_{i}\right)=u_{j}\left(a_{j}\right)\right)$ and the order-preserving property of $U\left(a_{1}, \ldots, a_{n}\right)=\sum_{i=1}^{n} u_{i}\left(a_{i}\right)$, it follows that

$$
\left(k a_{i}, e_{\bar{i}}\right) \sim\left(k a_{j}, e_{\bar{j}}\right)
$$

Equation 4 is similar to the concept of "law of exchange" in (Krantz et al., 1971, Definition 10.5). The law means the restriction for a 2-component conjoint structure with a multiplicative representation, ${ }^{6}$ say $U\left(a_{1}, a_{2}\right)=u_{1}\left(a_{1}\right) u_{2}\left(a_{2}\right)$, whose components are extensive structures with respect to either the induced conjoint order or its converse. A general form of Eq. 4 will be shown in the corollary to Lemma 2 in Sect. 5.

Finally, additional description is to be made in purport that the problem for the ordering of the joint effect is not completely excluded from the axiom system because of existence of cancellation. The cancellation axiom, however, is useful for extending the representation to $A^{(n)}$. In the meanwhile, the additive representation is, as will be

\footnotetext{
6 See Theorem 7.4 in Krantz et al. (1971) for an axiom system for the multiplicative representation. The axiom system has a generalized concept of independence of a stronger type, called sign dependence.
} 
dealt with in the proof of Theorem 2, constructed on $S^{(n)}$ disregarding the cancellation. It appears comparatively easy to acknowledge the single-factor independence (Matsushita 2007) and additive solvability on a small bounded domain. Therefore provided that weak ordering is accepted, the additive representation will turn out to be locally feasible.

\section{Conclusion}

This article axiomatized the additive representation on $A^{(n)}=\prod_{i=1}^{n} A_{i}$ on the assumption that each $A_{i}$ is a complete, continuous extensive structure. Attentive consideration might be disregarded with the ordering of the joint effect, based on the fact that the presented axiom system has the weaker independence. Additive solvability along with the weaker independence plays a key role in constructing the additive representation on a bounded subset. Likewise, the stronger solvability and cancellation play a key role in extending the representation to the whole set $A^{(n)}$. To apply the additive model to decision-making problems where a binary operation is possible on each component, empirical tests of additive solvability and cancellation are very important.

\section{Proofs}

\subsection{Proposition 1}

Proof (i) Assume that $c \succ a \circ b$. Since there is no minimal positive element, by A5, we take $x \in A$ greater than $e$ such that $c \succ(a \circ b) \circ x$. By A4 and A1, $c \succ a \circ(b \circ x)$, but here by $\mathrm{A} 7, b \circ x \succ b$.

(ii) If $b \sim e$, then the conclusion is trivial; so we consider the case where $b \succ e$. Assume that $a \succ b$, and let $X=\{x \in A \mid b \circ x$ is defined and $a \succ b \circ x\}$. By A5, $X$ is not empty. We provide two cases depending on whether $a$ is a maximal element or not.

Case 1. Element a is not maximal. Since there is $a^{\prime} \in A$ such that $a^{\prime} \succ a$, by A5, $a^{\prime} \succsim a \circ c$ for some $c \in A$. We show that there is a positive integer $n$ such that $a \circ c \succsim b \circ(n+1) c \succ a$. Assume that $a \succsim b \circ n c$ for all $n \in \mathbb{Z}^{+}$. Then, by A7, $a \succ n c$ for all $n$, in contradiction to A8. Therefore by letting $n=\sup \left\{k \in \mathbb{Z}^{+} \mid a \succsim b \circ k c\right\}$, we have $b \circ(n+1) c \succ a$, and by A3, $a \circ c \succsim b \circ(n+1) c$. The former inequality implies that $X$ is bounded above, and therefore by completeness, we can set $s=\sup X$. Here, $s$ must be $\prec(n+1) c$. Indeed, we see that $s \succ(n+1) c$ is impossible because $b \circ(n+1) c \succ a$. Moreover, if $s \sim(n+1) c$, then $b \circ s$ is defined. Therefore by lower semicontinuity, $b \circ x \succ a$ for $x \prec s$, in contradiction to the definition of $s$. Thus $s \prec(n+1) c$, and therefore, $b \circ s$ can be defined. It is clear from the definition of $s$ that $b \circ s \succsim a$. To verify that $b \circ s$ cannot be greater than $a$, assume contrarily to the above that $b \circ s \succ a$. Then, the argument referred to in this manner gives the same contradiction. Thus $a \sim b \circ s$.

Case 2. Element a is maximal. In the second half of this proof, we write the open interval determined by two points $a, c$ with $a \succ c$ as $(c, a)$, i.e., $(c, a)=\{y \in$ $A \mid c \prec y \prec a$ \}. Since $X$ is bounded above, let $s=\sup X$ again. Let $c \in A$ be an 
arbitrary element smaller than $a$, but larger than $b$, i.e., $a \succ c \succ b$. From the result of Case 1, we take $t \in A$ such that $c \sim b \circ t$. By A4 and A5, $a \succsim b \circ\left(t \circ t^{\prime}\right) \succ b \circ t$ for some $t^{\prime} \in A$. By A3, if $y \succ t$, then $b \circ y \succ c$. By the definition of $s$, if $s \succ y$, then $a \succ b \circ y$. Thus for any interval $(c, a)$, there exists an interval $(t, s)$ such that if $y \in(t, s)$, then $b \circ y \in(c, a)$; that is, $\lim _{y \uparrow s}(b \circ y) \sim b \circ\left(\lim _{y \uparrow s} y\right)$, where $y \uparrow s$ means that $y$ increases to $s$. Since $\lim _{y \uparrow s} y=s$ by A5, it suffices to show that the left-hand side is equal to $a$. Let $\lim _{y \uparrow s}(b \circ y)=a^{\prime}$, so that $a^{\prime} \sim b \circ s$. Now, assume that $a \succ a^{\prime}$. Then, by A5, $a \succ a^{\prime} \circ x$ for some $x \in A$. The result of Case 1 implies that $a^{\prime} \circ x \sim b \circ x^{\prime}$ for some $x^{\prime} \in A$. Hence $a \succ b \circ x^{\prime} \succ a^{\prime} \sim b \circ s$, so by A3, $x^{\prime} \succ s$, in contradiction to the definition of $s$. Thus we obtain $a \sim b \circ s$.

\subsection{Theorem 1}

Proof From Theorem 3.3 in Krantz et al. (1971), we obtain a real-valued function $u$ on $A$ satisfying Eqs. 1 and 2. Also from Eqs. 1 and 2, $u(e)=u(a \circ e)=u(a)+u(e)$. Thus $u(e)=0$ (Eq. 3). To prove the continuity of $u$, it suffices to show that $u$ has no gap in its range, because $u$ having no gap implies continuity of $u$ (see the proof of Theorem 19.5 in Krantz et al. (1990)). Suppose that $u(b)>u\left(b^{\prime}\right)$ for $b, b^{\prime} \in A$ so that $b \succ b^{\prime}$. Similarly to proof (i) of Proposition 1, we can take $c \in A$ such that $b \succ b^{\prime} \circ c$ and $b^{\prime} \succ c$. By A8, there is the largest integer $k \in \mathbb{Z}^{+}$such that $b^{\prime} \succsim k c$. This along with A3 implies that $b^{\prime} \circ c \succsim(k+1) c \succ b^{\prime}$. In view of the inequality $b \succ b^{\prime} \circ c$ and $\mathrm{A} 1, b \succ(k+1) c \succ b^{\prime}$. Therefore, by letting $x=(k+1) c, u(b)>u(x)>u\left(b^{\prime}\right)$ for some $x \in A$, as required.

\subsection{Preliminary lemmas}

Lemmas playing important roles in constructing the additive representation of Theorem 2 are hereby presented. The hypothesis of the following three lemmas is that $\left\langle A^{(n)}, \succsim\right\rangle$ is an additive conjoint structure on the product of extensive structures.

Lemma 1 Let $j \in N$ be an arbitrary index. There exists some $f_{j} \in A_{j}$ greater than $e_{j}$ such that for each $i \neq j$, we have an $f_{i} \in A_{i}$ satisfying $\left(f_{i}, e_{\bar{i}}\right) \sim\left(f_{j}, e_{\bar{j}}\right)$.

Proof First, choose an element $f_{i} \in A_{i}$ greater than $e_{i}$ for every $i \in N$. Then, the following step is repeated from $i=1$ to $n$ except for $i=j$. If $\left(f_{i}, e_{\bar{i}}\right) \succsim\left(f_{j}, e_{\bar{j}}\right)$, then we accept the element $f_{j}$. Otherwise Axiom 3 implies that since $\left(f_{j}, e_{j}\right) \succ$ $\left(f_{i}, e_{\bar{i}}\right)\left(\succ\left(e_{j}, e_{\bar{j}}\right)\right),\left(f_{j}^{\prime}, e_{\bar{j}}\right) \sim\left(f_{i}, e_{\bar{i}}\right)$ for some $f_{j}^{\prime} \in A_{j}$. Since $A_{j}$ has no minimal positive element, there is an $f_{j}^{\prime \prime} \in A_{j}$ with $f_{j}^{\prime} \succ_{j} f_{j}^{\prime \prime} \succ_{j} e_{j}$. Hence by Axiom 2, $\left(f_{j}^{\prime}, e_{\bar{j}}\right) \succ\left(f_{j}^{\prime \prime}, e_{\bar{j}}\right)$, so $\left(f_{i}, e_{\bar{i}}\right) \succsim\left(f_{j}^{\prime \prime}, e_{\bar{j}}\right)$. In this case, $f_{j}$ is replaced by $f_{j}^{\prime \prime}$. The procedure leads to $\left(f_{i}, e_{\bar{i}}\right) \succsim\left(f_{j}, e_{\bar{j}}\right)$ for all $i \neq j$. Finally, it is seen from Axiom 3 that there is an $f_{i}^{\prime} \in A_{i}$ such that $\left(f_{i}^{\prime}, e_{\bar{i}}\right) \sim\left(f_{j}, e_{\bar{j}}\right)$ for each $i \neq j$.

Lemma $2 \operatorname{Let}\left(f_{i}, e_{\bar{i}}\right) \sim\left(f_{j}, e_{\bar{j}}\right)$ for each $i \neq j$. If $c_{i} \circ_{i}\left(k_{i}+1\right) f_{i}, c_{j} \circ_{j} k_{j} f_{j}(j \neq i)$ are defined for $c_{i} \in A_{i}, c_{j} \in A_{j}$, and $k_{i}, k_{j} \in \mathbb{Z}_{0}^{+}$, then

$$
\left(c_{i} \circ_{i}\left(k_{i}+1\right) f_{i}, c_{j} \circ_{j}\left(k_{j}-1\right) f_{j}, c_{\overline{i j}} \circ_{\overline{i j}} k_{\overline{i j}} f_{\overline{i j}}\right) \sim\left(c_{i} \circ_{i} k_{i} f_{i}, c_{\bar{i}} \circ_{\bar{i}} k_{\bar{i}} f_{\bar{i}}\right),
$$


where $c_{\overline{i j}} \circ_{\overline{i j}} k_{\overline{i j}} f_{\overline{i j}}=\left(c_{1} \circ_{1} k_{1} f_{1}, \ldots, c_{i-1} \circ_{i-1} k_{i-1} f_{i-1}, c_{i+1} \circ_{i+1} k_{i+1} f_{i+1}, \ldots\right.$, $\left.c_{j-1} \circ_{j-1} k_{j-1} f_{j-1}, c_{j+1} \circ_{j+1} k_{j+1} f_{j+1}, \ldots, c_{n} \circ_{n} k_{n} f_{n}\right)$.

Proof We prove the lemma by using reductive absurdity. Suppose that $\left(\left(c_{i} \circ_{i} k_{i} f_{i}\right) \circ_{i}\right.$ $\left.f_{i}, c_{j} \circ_{j}\left(k_{j}-1\right) f_{j}, c_{\overline{i j}} \circ \overline{i j} k_{\overline{i j}} f_{\overline{i j}}\right) \succ\left(c_{i} \circ_{i} k_{i} f_{i}, c_{\bar{i}} \circ_{\bar{i}} k_{\bar{i}} f_{\bar{i}}\right)$. Then since $\left(c_{i} \circ_{i} k_{i} f_{i}, c_{\bar{i}} \circ_{\bar{i}}\right.$ $\left.k_{\bar{i}} f_{\bar{i}}\right) \succ\left(c_{i} \circ_{i} k_{i} f_{i}, c_{j} \circ_{j}\left(k_{j}-1\right) f_{j}, c_{\overline{i j}} \circ_{\overline{i j}} k_{\overline{i j}} f_{\overline{i j}}\right)$, it follows from Axiom 3 that there exists an $x_{i} \in A_{i}$ such that

$$
\left(\left(c_{i} \circ_{i} k_{i} f_{i}\right) \circ_{i} x_{i}, c_{j} \circ_{j}\left(k_{j}-1\right) f_{j}, c_{\overline{i j}} \circ_{\overline{i j}} k_{\overline{i j}} f_{\overline{i j}}\right) \sim\left(c_{i} \circ_{i} k_{i} f_{i}, c_{\bar{i}} \circ_{\bar{i}} k_{\bar{i}} f_{\bar{i}}\right) .
$$

Here, by Axiom 2, $x_{i} \prec_{i} f_{i}$ must be valid. Applying Axiom 4 inductively to the indifference, we have $\left(x_{i}, e_{\bar{i}}\right) \sim\left(f_{j}, e_{\bar{j}}\right)$. But since $\left(f_{i}, e_{\bar{i}}\right) \sim\left(f_{j}, e_{\bar{j}}\right)$, Axioms 1 and 2 imply that $x_{i} \sim_{i} f_{i}$, in contradiction to $x_{i} \prec_{i} f_{i}$. Next, suppose that $\left(\left(c_{i} \circ_{i} k_{i} f_{i}\right) \circ_{i} f_{i}, c_{j} \circ_{j}\left(k_{j}-1\right) f_{j}, c_{\overline{i j}} \circ_{\overline{i j}} k_{\overline{i j}} f_{\overline{i j}}\right) \prec\left(c_{i} \circ_{i} k_{i} f_{i}, c_{\bar{i}} \circ_{\bar{i}} k_{\bar{i}} f_{\bar{i}}\right)$. A similar argument implies the existence of $x_{j} \in A_{j}$ such that

$$
\begin{aligned}
& \left(c_{i} \circ_{i} k_{i} f_{i},\left(c_{j} \circ_{j}\left(k_{j}-1\right) f_{j}\right) \circ_{j} x_{j}, c_{\overline{i j}} \circ_{\overline{i j}} k_{\overline{i j}} f_{\overline{i j}}\right) \sim \\
& \quad\left(\left(c_{i} \circ_{i} k_{i} f_{i}\right) \circ_{i} f_{i}, c_{j} \circ_{j}\left(k_{j}-1\right) f_{j}, c_{\overline{i j}} \circ_{\overline{i j}} k_{\overline{i j}} f_{\overline{i j}}\right) .
\end{aligned}
$$

Here, by Axiom $2, x_{j} \prec_{j} f_{j}$. Again, the inductive application of Axiom 4 yields $\left(x_{j}, e_{\bar{j}}\right) \sim\left(f_{i}, e_{\bar{i}}\right)$, and therefore $x_{j} \sim_{j} f_{j}$, in contradiction to $x_{j} \prec_{j} f_{j}$. Thus, the conclusion is obtained from Axiom 1 .

Corollary 1 If $\left(f_{i}, e_{\bar{i}}\right) \sim\left(f_{j}, e_{\bar{j}}\right)(i \neq j)$ and $c_{i} \circ_{i} k_{i} f_{i}, c_{i} \circ_{i} k_{i}^{\prime} f_{i}$ are defined, then

$$
\left(c_{i} \circ_{i} k_{i} f_{i}, c_{j} \circ_{j} k_{\bar{i}} f_{\bar{i}}\right) \sim\left(c_{i} \circ_{i} k_{i}^{\prime} f_{i}, c_{j} \circ_{j} k_{\bar{i}}^{\prime} f_{\bar{i}}\right) \text { whenever } \sum_{i=1}^{n} k_{i}=\sum_{i=1}^{n} k_{i}^{\prime} .
$$

In particular, Eq. 4 in Sect. 3 is satisfied:

$$
\left(k f_{i}, e_{i}^{-}\right) \sim\left(k f_{j}, e_{\bar{j}}\right) \text { for } k \in \mathbb{Z}^{+} .
$$

Lemma 3 There exist additive representations $u_{i}$ on $A_{i}$ and $u_{j}$ on $A_{j}(i \neq j)$ such that

$$
u_{i}\left(a_{i}\right)=u_{j}\left(a_{j}\right) \text { whenever }\left(a_{i}, e_{\bar{l}}\right) \sim\left(a_{j}, e_{\bar{j}}\right) .
$$

Proof For any $a_{i} \succsim_{i} x_{i} \succ_{i} e_{i}$, let $N\left(x_{i}, a_{i}\right)$ denote the largest positive integer such that $N\left(x_{i}, a_{i}\right) x_{i}$ is defined and $a_{i} \succsim_{i} N\left(x_{i}, a_{i}\right) x_{i}$. Let $\left\{x_{i}^{(m)}\right\}_{m=1,2, \ldots}$ be a sequence in $A_{i}$ such that $x_{i}^{(m)} \succsim_{i} 2 x_{i}^{(m+1)}$ for all $m \in \mathbb{Z}^{+}$(see the proof of Theorem 2.4 in Krantz et al. (1971)). Then since $x_{i}^{(m)} \succsim_{i} 2^{k} x_{i}^{(m+k)}$ for all $k \geq 0$, it follows from the Archimedean property that for any positive $c_{i} \in A_{i}, c_{i} \succ_{i} x_{i}^{(m)}$ for all but finitely many values of $m$, in which case we say that $\left\{x_{i}^{(m)}\right\}_{m=1,2, \ldots}$ converges to zero. By using the construction method given in the proof of Krantz et al., define a function 
$u_{i}$ on $A_{i}$ as $u_{i}\left(a_{i}\right)=\lim _{m \rightarrow \infty} N\left(x_{i}^{(m)}, a_{i}\right) / N\left(x_{i}^{(m)}, f_{i}\right)$, where $f_{i} \succ_{i} e_{i}$ is a fixed element and is interpreted as a unit, i.e., $u_{i}\left(f_{i}\right)=1$. We will also construct a sequence in $A_{j}$ that converges to zero. First, take an $x_{j}^{(1)} \in A_{j}$ such that $\left(x_{j}^{(1)}, e_{\bar{j}}\right) \sim\left(x_{i}^{(1)}, e_{\bar{i}}\right)$ by Lemma 1 . Next, let $x_{j}^{(1)}, \ldots, x_{j}^{(m)}$ be such that $\left(x_{j}^{(k)}, e_{\bar{j}}\right) \sim\left(x_{i}^{(k)}, e_{\bar{i}}\right)$ and such that $x_{j}^{(k)} \succsim_{j} 2 x_{j}^{(k+1)}$ for all $k<m$. Recall $x_{i}^{(m)} \succsim_{i} 2 x_{i}^{(m+1)}$. Then since $\left(x_{j}^{(m)}, e_{j}\right) \succ$ $\left(x_{i}^{(m+1)}, e_{\bar{i}}\right) \succ\left(e_{j}, e_{\bar{j}}\right)$ by Axioms 1 and 2 , Axiom 3 implies the existence of an $x_{j}^{(m+1)} \in A_{j}$ such that $\left(x_{j}^{(m+1)}, e_{j}^{-}\right) \sim\left(x_{i}^{(m+1)}, e_{i}^{-}\right)$. By Eq. 4 and Axioms 1 and 2 , $\left(x_{j}^{(m)}, e_{\bar{j}}\right) \succsim\left(2 x_{j}^{(m+1)}, e_{\bar{j}}\right)$, so by Axiom $2, x_{j}^{(m)} \succsim_{j} 2 x_{j}^{(m+1)}$. Hence the sequence $\left\{x_{j}^{(m)}\right\}_{m=1,2, \ldots}$ converges to zero. Let $\left(a_{i}, e_{i}\right) \sim\left(a_{j}, e_{j}\right)$. We shall now acknowledge that $N\left(x_{i}^{(m)}, a_{i}\right) x_{j}^{(m)}$ is defined. Then for every $m$,

$$
\begin{aligned}
a_{i} \succsim_{i} N\left(x_{i}^{(m)}, a_{i}\right) x_{i}^{(m)} & \Leftrightarrow\left(a_{i}, e_{\bar{i}}\right) \succsim\left(N\left(x_{i}^{(m)}, a_{i}\right) x_{i}^{(m)}, e_{\bar{i}}\right) & & \text { (Axiom 2) } \\
& \Leftrightarrow\left(a_{j}, e_{\bar{j}}\right) \succsim\left(N\left(x_{i}^{(m)}, a_{i}\right) x_{j}^{(m)}, e_{\bar{j}}\right) & & \text { (Axiom 1, Eq. 4) } \\
& \Leftrightarrow a_{j} \succsim_{j} N\left(x_{i}^{(m)}, a_{i}\right) x_{j}^{(m)} & & \text { (Axiom 2) }
\end{aligned}
$$

So by definition, $N\left(x_{j}^{(m)}, a_{j}\right) \geq N\left(x_{i}^{(m)}, a_{i}\right)$. By interchanging the roles of $N\left(x_{i}^{(m)}, a_{i}\right)$ and $N\left(x_{j}^{(m)}, a_{j}\right)$, we have $N\left(x_{i}^{(m)}, a_{i}\right) \geq N\left(x_{j}^{(m)}, a_{j}\right)$. Hence $N\left(x_{i}^{(m)}, a_{i}\right)=$ $N\left(x_{j}^{(m)}, a_{j}\right)$. Since we may assume that $\left(f_{i}, e_{\bar{i}}\right) \sim\left(f_{j}, e_{\bar{j}}\right)$ by Lemma 1 , a similar method gives $N\left(x_{i}^{(m)}, f_{i}\right)=N\left(x_{j}^{(m)}, f_{j}\right)$. For simplicity, set $y_{i}^{(m)}=N\left(x_{i}^{(m)}, a_{i}\right) /$ $N\left(x_{i}^{(m)}, f_{i}\right), y_{j}^{(m)}=N\left(x_{j}^{(m)}, a_{j}\right) / N\left(x_{j}^{(m)}, f_{j}\right)$. Let $\lim _{m \rightarrow \infty} y_{i}^{(m)}=\alpha, \lim _{m \rightarrow \infty} y_{j}^{(m)}$ $=\alpha^{\prime}$, where $\alpha, \alpha^{\prime} \in \mathbb{R}_{0}^{+}$(recall that the limits of $y_{i}^{(m)}, y_{j}^{(m)}$ are definite). Therefore given $\varepsilon_{1}>0$, there is an integer $N_{1}$ such that $\left|y_{j}^{(k)}-\alpha^{\prime}\right|<\varepsilon_{1}$ whenever $k \geq N_{1}$. Since $\left\{y_{j}^{(m)}\right\}$ is a Cauchy sequence, given $\varepsilon_{2}>0$, there is an integer $N_{2}$ such that $\left|y_{j}^{(k)}-y_{j}^{(l)}\right|<\varepsilon_{2}$ whenever $l>k \geq N_{2}$. Let $N=\max \left\{N_{1}, N_{2}\right\}$. In view of the fact that $y_{j}^{(k)}=y_{i}^{(k)}$ for each $k$, it follows that $\left|y_{i}^{(k)}-\alpha^{\prime}\right| \leq\left|y_{j}^{(k)}-y_{j}^{(l)}\right|+\left|y_{j}^{(l)}-\alpha^{\prime}\right|<$ $\varepsilon_{1}+\varepsilon_{2}$ whenever $l>k \geq N$. Thus $\alpha=\alpha^{\prime}$.

Lemma 4 Let $F_{i}(i=1, \ldots, n)$ be a real-valued function on $A_{\bar{i}}^{(n)}$. If

$$
u_{1}\left(a_{1}\right)+F_{1}\left(a_{\overline{1}}\right)=\cdots=u_{n}\left(a_{n}\right)+F_{n}\left(a_{\bar{n}}\right),
$$

then for each $i=1, \ldots, n, F_{i}\left(a_{\bar{i}}\right)=\sum_{k \neq i} u_{k}\left(a_{k}\right)$.

Proof By Eq. 3, $u_{i}\left(e_{i}\right)=0$ for each $i=1, \ldots, n$. Substituting $a_{1}=e_{1}, \ldots, a_{n}=e_{n}$ in Eq. 6 yields

$$
F_{1}\left(e_{\overline{1}}\right)=\cdots=F_{n}\left(e_{\bar{n}}\right)
$$

Without loss of generality, set $F_{i}\left(e_{\bar{i}}^{-}\right)=0$ since, if necessary, we may subtract a constant from each side of Eq. 6. Let $N(j)=\{1, \ldots, j-1, j+1, \ldots, n\}$ and 
$I=\left\{i_{1}, \ldots, i_{k}\right\}\left(i_{1}<\cdots<i_{k}\right)$ with $I \subseteq N(j)$. Let $a_{N(j), I}$ be an element $\left(a_{\bar{j}}\right) \in A_{\bar{j}}^{(n)}$ such that

$$
a_{i} \neq e_{i} \text { if } i \in I \text { and } a_{i}=e_{i} \text { if } i \in N(j)-I .
$$

Then, $k=|I|$ is the number of indexes $i$ such that $a_{i} \neq e_{i}$. By induction on $k$, we prove the lemma. First, substituting $a_{i}=e_{i}\left(i \neq i_{1}\right)$ in Eq. 6, we have

$$
F_{j}\left(a_{N(j),\left\{i_{1}\right\}}\right)=u_{i_{1}}\left(a_{i_{1}}\right) .
$$

Therefore, Lemma 4 is valid if $k=1$ because $j$ is arbitrary. Next, assume that for any $j=1, \ldots, n$, if $|I|=k$, then

$$
F_{j}\left(a_{N(j), I}\right)=u_{i_{1}}\left(a_{i_{1}}\right)+\cdots+u_{i_{k}}\left(a_{i_{k}}\right) .
$$

Let $I^{\prime}=\left\{i_{1}, \ldots, i_{k+1}\right\}\left(i_{1}<\cdots<i_{k+1}\right)$ with $I^{\prime} \subseteq N(j)$. Substituting $a_{j}=e_{j}$ and $a_{i}=e_{i}\left(i \in N(j)-I^{\prime}\right)$ in Eq. 6, we have

$$
F_{j}\left(a_{N(j), I^{\prime}}\right)=u_{i_{k+1}}\left(a_{i_{k+1}}\right)+F_{i_{k+1}}\left(a_{N\left(i_{k+1}\right), I}\right) .
$$

By the inductive assumption,

$$
F_{j}\left(a_{N(j), I^{\prime}}\right)=u_{i_{1}}\left(a_{i_{1}}\right)+\cdots+u_{i_{k}}\left(a_{i_{k}}\right)+u_{i_{k+1}}\left(a_{i_{k+1}}\right) .
$$

Clearly, this equation holds for any $j=1, \ldots, n$. This proves Lemma 4 .

\subsection{Theorem 2}

If an element $\left(a_{i}, a_{\bar{i}}\right)$ exists between $\left(\bar{b}_{i}, b_{\bar{i}}\right)$ and $\left(\underline{b}_{i}, b_{\bar{i}}\right)$, then we can define the image of $a_{\bar{i}}, b_{\bar{i}}$ on $A_{i}, \varphi_{i}\left(a_{\bar{i}}, b_{\bar{i}}\right)$, as the solution to $\left(a_{i} \circ_{i} \varphi_{i}\left(a_{\bar{i}}, b_{\bar{i}}\right), b_{\bar{i}}\right) \sim\left(a_{i}, a_{\bar{i}}\right)$. Since $\succsim$ is single-factor independent and $A_{i}$ is an extensive structure, it is seen that $\varphi_{i}\left(a_{\bar{i}}, b_{\bar{i}}\right)$ is unique up to $\sim_{i}$. Since we consider the image keeping $b_{\bar{i}}$ fixed at $e_{\bar{i}}$ throughout the following proof, we can write $\varphi_{i}\left(a_{\bar{i}}, e_{\bar{i}}\right)=\varphi_{i}\left(a_{\bar{i}}\right)$. The proof is divided into four parts. That is, the first part guarantees the existence of a symmetrically bounded set from above $S^{(n)}$; the second constructs the representation on $S^{(n)}$; the third extends the result to all of $A^{(n)}$; and the fourth proves its uniqueness.

Proof Assume that Axioms 1-4 of Definition 6 hold. It is easily seen that $A^{(n)}$ includes a symmetrically bounded set from above. For a fixed $j \in N$, take an element $\bar{f}_{j j} \in A_{j}$ greater than $e_{j}$ such that $n \bar{f}_{j j}$ is defined (such $\bar{f}_{j j}$ is certain to exist, as was stated in the proof of Lemma3). Using Lemma 1, we find an element $\bar{f}_{i j} \in A_{i}$ such that $\left(\bar{f}_{i j}, e_{\bar{i}}\right) \sim\left(\bar{f}_{j j}, e_{\bar{j}}\right)$ for each $i \neq j$. This is done for all $j \in N$; set $\bar{f}_{i}=\min _{j \in N}\left\{\bar{f}_{i j}\right\}$ for each $i \in N$. Since all $\bar{f}_{i}(i \in N)$ are chosen from $\left\{\bar{f}_{i j}\right\}$ with a common $j$, it is valid that $\left(\bar{f}_{i}, e_{\bar{i}}\right) \sim\left(\bar{f}_{j}, e_{\bar{j}}\right)$ for each $i \neq j$. It 
is clear that the set of $\left(a_{1}, \ldots, a_{n}\right)$ such that $\bar{f}_{i} \succsim_{i} a_{i} \succsim_{i} e_{i}$ is a required symmetrically bounded set from above. But, for the proof of the extension part, we define $S^{(n)}=\left\{\left(a_{1}, \ldots, a_{n}\right) \mid 2 f_{i} \succsim_{i} a_{i} \succsim_{i} e_{i}\right.$ and $2 n f_{i}$ is defined for $\left.i=1, \ldots, n\right\}$, where $f_{i}$ is regarded as a unit element. The definition is feasible because whenever $\left(f_{i}, e_{\bar{i}}\right) \sim\left(f_{j}, e_{\bar{j}}\right)$, then by Eq. $4,\left(2 f_{i}, e_{\bar{i}}\right) \sim\left(2 f_{j}, e_{\bar{j}}\right)$. The indifference statements $\left(f_{i}, f_{\bar{i}}\right) \sim\left(n f_{i}, e_{\bar{j}}\right)$ and $\left(2 f_{i}, 2 f_{\bar{i}}\right) \sim\left(2 n f_{i}, e_{\bar{j}}\right)$ are also valid by Eq. 5 . These will be used in applying restricted solvability.

We construct the additive representation on $S^{(n)}$. Let $a=\left(a_{i}, a_{\bar{i}}\right) \in S^{(n)}$ and $b=\left(b_{i}, b_{\bar{i}}\right) \in S^{(n)}$. Since $\left(2 n f_{i}, e_{\bar{i}}\right) \succsim a, b \succsim\left(e_{i}, e_{\bar{i}}\right)$, by Axiom 3 , we have $a \sim\left(a_{i} \circ_{i} \varphi_{i}\left(a_{\bar{i}}\right), e_{\bar{i}}\right)$ and $b \sim\left(b_{i} \circ_{i} \varphi_{i}\left(b_{\bar{i}}\right), e_{\bar{i}}\right)$. Hence,

$$
\begin{aligned}
a \succsim b & \left.\Leftrightarrow\left(a_{i} \circ_{i} \varphi_{i}\left(a_{\bar{i}}\right), \quad e_{\bar{i}}\right) \succsim_{\left(b_{i} \circ_{i}\right.} \varphi_{i}\left(b_{\bar{i}}\right), e_{\bar{i}}\right) \\
& \Leftrightarrow a_{i} \circ_{i} \varphi_{i}\left(a_{\bar{i}}\right) \succsim_{i} b_{i} \circ_{i} \varphi_{i}\left(b_{\bar{i}}\right) \\
& \Leftrightarrow u_{i}\left(a_{i} \circ_{i} \varphi_{i}\left(a_{\bar{i}}\right)\right) \geq u_{i}\left(b_{i} \circ_{i} \varphi_{i}\left(b_{\bar{i}}\right)\right)
\end{aligned}
$$

To construct the additive representation, it is sufficient to show that $u_{i}\left(a_{i} \circ_{i} \varphi_{i}\left(a_{\bar{i}}\right)\right)=$ $\sum_{i=1}^{n} u_{i}\left(a_{i}\right)$. Since $\left(a_{i} \circ_{i} \varphi_{i}\left(a_{\bar{i}}\right), e_{\bar{i}}\right) \sim\left(a_{j} \circ_{j} \varphi_{j}\left(a_{\bar{j}}\right), e_{\bar{j}}\right)$ for all $i \neq j$ by Axiom 3 , it follows from Lemma 3 that $u_{i}\left(a_{i} \circ_{i} \varphi_{i}\left(a_{\bar{i}}\right)\right)=u_{j}\left(b_{j} \circ_{j} \varphi_{j}\left(b_{\bar{j}}\right)\right)$. Setting $F_{i}\left(a_{\bar{i}}\right)=$ $u_{i}\left(\varphi_{i}\left(a_{\bar{i}}\right)\right)$, we have $u_{i}\left(a_{i}\right)+F_{i}\left(a_{\bar{i}}\right)=u_{j}\left(a_{j}\right)+F_{j}\left(a_{\bar{j}}\right)$. Hence, Lemma 4 provides the additive form required above.

Next, we extend the additive representation on $S^{(n)}$ to $A^{(n)}$. Let $a=\left(a_{i}, a_{\bar{i}}\right) \in A^{(n)}$. The Archimedean property of $A_{i}$ implies the existence of the largest integer $k_{i} \in \mathbb{Z}_{0}^{+}$ such that $a_{i} \succsim_{i} k_{i} f_{i}$. By Proposition $1, a_{i} \sim_{i} k_{i} f_{i} \circ_{i} c_{i}$ for some $c_{i} \in A_{i}$, where $c_{i} \prec_{i} f_{i}$. Repeated use of Axiom 2 gives $\left(a_{i}, a_{\bar{i}}^{-}\right) \sim\left(k_{i} f_{i} \circ_{i} c_{i}, k_{\bar{i}} f_{\bar{i}} \circ_{\bar{i}} c_{\bar{i}}\right)$. Since $\left(f_{i}, f_{\bar{i}}\right) \succ\left(c_{i}, c_{\bar{i}}\right) \succsim\left(e_{i}, e_{\bar{i}}\right)$ and $\left(f_{i}, f_{\bar{i}}\right) \sim\left(n f_{i}, e_{\bar{i}}\right)$, by Axiom 3 ,

$$
\left(c_{i}, c_{\bar{i}}\right) \sim\left(c_{i} \circ_{i} \varphi_{i}\left(c_{\bar{i}}\right), e_{\bar{i}}\right)
$$

In view of the result $u_{i}\left(c_{i} \circ_{i} \varphi_{i}\left(c_{\bar{i}}^{-}\right)\right)=\sum_{i=1}^{n} u_{i}\left(c_{i}\right)$ (the second paragraph), let a function $U$ on $A^{(n)}$ be defined as

$$
U\left(a_{i}, a_{\bar{i}}\right)=\sum_{i=1}^{n} u_{i}\left(c_{i}\right)+\sum_{i=1}^{n} k_{i} .
$$

Since $u_{i}\left(f_{i}\right)=1$ and $u_{i}\left(a_{i}\right)=u_{i}\left(c_{i}\right)+u_{i}\left(k_{i} f_{i}\right)$, it follows from Eq. 7 that $U\left(a_{i}, a_{\bar{i}}\right)=$ $\sum_{i=1}^{n} u_{i}\left(a_{i}\right)$.

Henceforth, we show that $U$ preserves $\succsim$. Let $b=\left(b_{i}, b_{\bar{i}}\right)$ be another element of $A^{(n)}$, and let $l_{i} \in \mathbb{Z}_{0}^{+}$be the largest integer such that $b_{i} \succsim_{i} l_{i} f_{i}$, so that $\left(b_{i}, b_{\bar{i}}\right) \sim$ $\left(l_{i} f_{i} \circ_{i} d_{i}, l_{\bar{i}} f_{\bar{i}} \circ_{\bar{i}} d_{\bar{i}}\right)$ for some $d_{i} \prec_{i} f_{i}$. Without loss of generality, assume $\sum_{i=1}^{n} k_{i} \geq$ $\sum_{i=1}^{n} l_{i}$. We now have two cases to consider.

Case 1. $\sum_{i=1}^{n} k_{i} \geq \sum_{i=1}^{n} l_{i}+n$. If necessary, using Eq. 5, we let $l_{i}^{\prime} \in \mathbb{Z}_{0}^{+}$be such that $\left(l_{i}^{\prime} f_{i} \circ_{i} d_{i}, l_{\bar{i}}^{\prime} f_{\bar{i}} \circ_{\bar{i}} d_{\bar{i}}\right) \sim\left(l_{i} f_{i} \circ_{i} d_{i}, l_{\bar{i}} f_{\bar{i}} \circ_{\bar{i}} d_{\bar{i}}\right)$ and $\left(l_{i}^{\prime}+1\right) f_{i}$ is defined for each $i$. This is feasible because $\sum_{i=1}^{n} k_{i} \geq \sum_{i=1}^{n} l_{i}+n$. Then, we can take $k_{i}^{\prime} \geq l_{i}^{\prime}+1$ for each $i$ such that $\sum_{i=1}^{n} k_{i}^{\prime}=\sum_{i=1}^{n} k_{i}$, so by Eq. 5, $\left(k_{i} f_{i}, k_{\bar{i}} f_{\bar{i}}\right) \sim\left(k_{i}^{\prime} f_{i}, k_{\bar{i}}^{\prime} f_{\bar{i}}\right)$. Since $k_{i}^{\prime} f_{i} \succ_{i} l_{i}^{\prime} f_{i} \circ_{i} d_{i}$, repeated use of Axiom 2 gives $\left(k_{i}^{\prime} f_{i}, k_{\bar{i}}^{\prime} f_{\bar{i}}\right) \succ\left(l_{i}^{\prime} f_{i} \circ_{i} d_{i}, l_{\bar{i}}^{\prime} f_{\bar{i}} \circ_{\bar{i}} d_{\bar{i}}\right)$. 
Hence, by Axiom 1, $a \succ b$. But since $\sum_{i=1}^{n} u_{i}\left(d_{i}\right)<n$, it is seen from Eq. 7 that $U(a)>U(b)$.

Case 2. $\sum_{i=1}^{n} k_{i}<\sum_{i=1}^{n} l_{i}+n$. Three cases can be considered according to the preference patterns arising between $a$ and $b$.

Case 2A. $a \sim b$. By Eq. 5 and inductive use of Axiom 4, we have $\left(k_{i}^{\prime} f_{i} \circ_{i} c_{i}, k_{\bar{i}}^{\prime} f_{\bar{i}} \circ_{\bar{i}}\right.$ $\left.c_{\bar{i}}^{-}\right) \sim\left(d_{i}, d_{\bar{i}}\right)$ where $\sum_{i=1}^{n} k_{i}^{\prime}+\sum_{i=1}^{n} l_{i}=\sum_{i=1}^{n} k_{i}$. From the result in the second paragraph, it follows that

$$
\left(k_{i}^{\prime} f_{i} \circ_{i} c_{i}, k_{\bar{i}}^{\prime} f_{\bar{i}} \circ_{\bar{i}} c_{\bar{i}}\right) \sim\left(d_{i}, d_{\bar{i}}\right) \Leftrightarrow \sum_{i=1}^{n} u_{i}\left(k_{i}^{\prime} f_{i} \circ_{i} c_{i}\right)=\sum_{i=1}^{n} u_{i}\left(d_{i}\right)
$$

By adding $\sum_{i=1}^{n} l_{i}$ to both sides of the equality and from Eq. 7, it is seen that $U(a)=$ $U(b)$.

Case 2B. $a \succ b$. The proof is further divided into two cases:

(i) $a_{i} \precsim_{i} n f_{i}$ for all $i$. Since $a \succ b, b_{i}$ must be $\prec_{i} a_{i}$ for at least one $i$. First, if $\left(b_{i} \circ_{i}\right.$ $\left.n f_{i}, b_{\bar{i}}\right) \succsim\left(a_{i}, a_{\bar{i}}\right)$, then by restricted solvability (Axiom 3$),\left(x_{i}, b_{\bar{i}}\right) \sim\left(a_{i}, a_{\bar{i}}\right)$ for some $x_{i} \in A_{i}$ with $x_{i} \succ_{i} b_{i}$. Hence, by Case 2A, $\sum_{i=1}^{n} u_{i}\left(a_{i}\right)=u_{i}\left(x_{i}\right)+$ $\sum_{j \neq i} u_{j}\left(b_{j}\right)>\sum_{i=1}^{n} u_{i}\left(b_{i}\right)$. Next, if $\left(a_{i}, a_{\bar{i}}\right) \succ\left(b_{i} \circ_{i} n f_{i}, b_{\bar{i}}\right)$, then $b_{j} \prec_{j} a_{j}$ must be valid for at least another one index $j \neq i$. But it is evident from the result of Case 1 (i.e., $\left.\sum_{i=1}^{n} l_{i}+2 n \geq \sum_{i=1}^{n} k_{i}+n\right)$ that $\left(b_{i} \circ_{i} n f_{i}, b_{j} \circ_{j} n f_{j}, b_{\overline{i j}}\right) \succ$ $\left(a_{i}, a_{\bar{i}}\right)$. Hence, by restricted solvability, $\left(b_{i} \circ_{i} n f_{i}, x_{j}, b_{\overline{i j}}\right) \sim\left(a_{i}, a_{\bar{i}}\right)$ for some $x_{j} \in A_{j}$ with $x_{j} \succ_{i} b_{i}$ so that $\sum_{i=1}^{n} u_{i}\left(a_{i}\right)=u_{j}\left(x_{j}\right)+\sum_{i \neq j} u_{i}\left(b_{i}\right)+n>$ $\sum_{i=1}^{n} u_{i}\left(b_{i}\right)$.

(ii) $a_{i} \succ_{i} n f_{i}$ for some $i$. First, if $\left(b_{i}, b_{\bar{i}}\right) \succsim\left(e_{i}, a_{\bar{i}}\right)$, then by restricted solvability, $\left(x_{i}, a_{\bar{i}}\right) \sim\left(b_{i}, b_{\bar{i}}\right)$ for some $x_{i} \in A_{i}$ with $x_{i} \prec_{i} a_{i}$. Hence $\sum_{i=1}^{n} u_{i}\left(a_{i}\right)>$ $u_{i}\left(x_{i}\right)+\sum_{j \neq i} u_{j}\left(a_{j}\right)=\sum_{i=1}^{n} u_{i}\left(b_{i}\right)$. Next, if $\left(e_{i}, a_{\bar{i}}\right) \succ\left(b_{i}, b_{\bar{i}}\right)$, then we again consider two cases: Case (i) ${ }^{\prime} . a_{j} \precsim j n f_{j}$ for all $j \neq i$; Case(ii)'. $a_{j} \succ_{j} n f_{j}$ for some $j \neq i$. In the former case, the result of Case (i) implies that $\sum_{j \neq i} u_{j}\left(a_{j}\right)>\sum_{i=1}^{n} u_{i}\left(b_{i}\right)$. In the latter case, it is evident from the result of Case 1 that $\left(b_{i}, b_{\bar{i}}\right) \succ\left(e_{i}, e_{j}, a_{\overline{i j}}\right)$. Hence, a similar method to the first half of Case (ii) gives $\sum_{i=1}^{n} u_{i}\left(a_{i}\right)>\sum_{i=1}^{n} u_{i}\left(b_{i}\right)$.

Case 2C. $a \prec b$. Consider two cases by replacing $a_{i}$ with $b_{i}$ in Cases 2B(i) and 2B(ii). However, from the result of Case $1,\left(a_{i} \circ_{i} n f_{i}, a_{\bar{i}}\right) \succsim\left(b_{i}, b_{\bar{i}}\right)$ and $\left(a_{i}, a_{\bar{i}}\right) \succsim$ $\left(e_{i}, b_{\bar{i}}\right)$ must be satisfied in the two respective cases. Hence, we can easily obtain the required result $U(a)<U(b)$.

Finally, we prove the uniqueness assertion. Assume that each $v_{i}$ is an additive representation on $A_{i}$ such that $v_{i}\left(e_{i}\right)=0$, and let $V(a)=\sum_{i=1}^{n} v_{i}\left(a_{i}\right)$. Hence $v_{i}=\alpha_{i} u_{i}$ for $\alpha_{i}>0$ (Theorem 1). If $\alpha_{i}=\alpha>0$, then $V$ preserves $\succsim$ because $V(a)=\alpha U(a)$. Conversely, assume that $V(a)$ is an order-preserving function in $\succsim$. Let $a_{(i, j)}(i \neq j)$ denote an element $\left(a_{1}, \ldots, a_{n}\right) \in A^{(n)}$ such that $a_{k}=e_{k}$ unless $k=i$ or $k=j$. Let $a_{(i, j)}, b_{(i, j)} \in A^{(n)}$ be such that $a_{(i, j)} \sim b_{(i, j)}$, with $a_{i}, a_{j}$ not indifferent to $b_{i}, b_{j}$, respectively, which is feasible by Axiom 3. From the equalities $U\left(a_{(i, j)}\right)=U\left(b_{(i, j)}\right)$ and $V\left(a_{(i, j)}\right)=V\left(b_{(i, j)}\right)$, we obtain 


$$
1=\frac{u_{j}\left(b_{j}\right)-u_{j}\left(a_{j}\right)}{u_{i}\left(a_{i}\right)-u_{i}\left(b_{i}\right)}=\frac{\alpha_{i}}{\alpha_{j}} .
$$

Since this is true for all distinct $i, j \in N$, we see that $\alpha_{i}=\alpha$ for all $i \in N$.

Acknowledgements I am grateful to the anonymous reviewers for helpful comments and suggestions.

\section{References}

Candeal, J. C., De Miguel, J. R., \& Induráin, E. (1996). Extensive measurement: Continuous additive utility functions on semigroups. Journal of Mathematical Psychology, 40, 281-286.

Fishburn, P. C. (1982). The foundations of expected utility. Dordrecht: Reidel.

Fuchs, L. (1963). Partially ordered algebraic systems. Reading, Massachusetts: Addison-Wesley.

Kahneman, D., \& Tversky, A. (1979). Prospect theory: An analysis of decision under risk. Econometrica, 47, 263-291.

Kelly, J. L. (1955). General topology. Princeton, NJ: Van Nostrand.

Krantz, D. H., Luce, R. D., Suppes, P., \& Tversky, A. (1971). Foundations of measurement (Vol. 1). New York: Academic Press.

Krantz, D. H., Luce, R. D., Suppes, P., \& Tversky, A. (1990). Foundations of measurement (Vol. 3). New York: Academic Press.

Luce, R. D. (1990). Rational versus plausible accounting equivalences in preference judgments. Psychological Science, 1, 225-234.

Luce, R. D. (2000). Utility of gains and losses: measurement-theoretical and experimental approaches. Mahwah, NJ: Erlbaum.

Luce, R. D. (2004). Symmetric and asymmetric matching of joint presentations. Psychological Review, 111, 446-454.

Marchant, T., \& Luce, R. D. (2003). Technical note on the joint receipt of quantities of a single good, Journal of Mathematical Psychology, 47, 66-74.

Matsushita, Y. (2007). A joint-receipt conjoint structure and its additive representation. Journal of Advanced Computational Intelligence and Intelligent Informatics, 11, 891-896.

Roberts, F. S., \& Luce, R. D. (1968). Axiomatic thermodynamics and extensive measurement. Synthese, 18, 311-326.

Wakker, P. P. (1989). Additive representations of preferences. Dordrecht: Kluwer. 